\title{
Modificación de conductas prono-coronarias en pacientes de estatus socio-económico bajo
}

\author{
Antonio del Pino* \\ M. ${ }^{\text {a Teresa Gaos* }}$ \\ Ruth Dorta* \\ Martín García** \\ * Faculty of Psychology. La Laguna \\ University. \\ ** Service of Cardiology. La Laguna \\ University Hospital.
}

SPAIN

\begin{abstract}
RESUMEN - Este trabajo presenta la efectividad de un tratamiento cognitivo-conductual aplicado en grupo. Participaron 98 varones afectos de enfermedad coronaria. Fueron asignados al azar a un tratamiento psicológico (TPs), a un programa de educación para la salud (PES) o a sólo el tratamiento médico estándar (TME) para pacientes coronarios. Después del año de tratamiento, sólo en el grupo con TPs se dieron diferencias estadísticamente significativas en Patrón de Conducta Tipo A (PCTA) medido por la Entrevista Estructurada (EE), y en ansiedad rasgo. Tras dos años de seguimiento, el grupo con TPs mostró una reducción estadísticamente significativa en PCTA, especialmente "RapidezImpaciencia”, y en depresión. Los resultados PCTA se valoran como un primer paso fiable en el proceso de validar este tratamiento que, además, ha conseguido reducir de forma significativa la ansiedad y depresión, un resultado no frecuente en los tratamientos psicoeducativos aplicados a pacientes coronarios.
\end{abstract}

\section{Introducción}

Los resultados de las intervenciones psicológicas realizadas hasta hace unos quince años con el propósito de cambiar factores de riesgo psico-social fueron recogidos en el meta-análisis de Nunes, Frank y Kornfeld (1987). Los autores concluyeron que la inter- vención psicológica había permitido reducir el Patrón de Conducta Tipo A (PCTA). El tamaño del efecto del cambio de PCTA varió entre 0,02 y 1,27 con una media de 0,61 , sin que ninguna modalidad única de tratamiento resultara efectiva. Sin embargo, los resultados mostraron que los estudios que utilizaron más modalidades de tratamiento lograron mayores 
tamaños del efecto que los que hicieron uso de menos tratamientos, y que la combinación de un componente educativo [bien sobre la enfermedad coronaria (EC) o bien sobre el PCTA], un método de afrontamiento o terapia cognitiva y un componente de ensayo conductual logró los coeficientes de correlación más altos con los cambios en PCTA.

Linden, Stossel y Maurice (1996) realizaron otro meta-análisis que evaluó el impacto de los tratamientos psicológicos sobre la rehabilitación de la EC documentada y encontraron que en los pacientes que recibieron un tratamiento psico-social junto con la rehabilitación cardiaca estándar hubo una reducción de la mortalidad y la morbilidad, especialmente durante los dos primeros años. El meta-análisis de Dusseldorp et al. (1999) examinó los efectos de los programas psico-educativos (educación sanitaria y manejo del estrés) con pacientes coronarios. Los resultados sugirieron efectos positivos a largo plazo (reducción del 34\% en mortalidad cardíaca y del $29 \%$ en recurrencia de infarto de miocardio) y efectos positivos significativos en objetivos próximos (presión sanguínea sistólica, colesterol, conducta de fumar, etc.). No se encontraron efectos de los programas psico-educativos con relación a la ansiedad y la depresión.

Los autores de estos meta-análisis expresan la conveniencia de adaptar los programas a las características de los diferentes grupos de pacientes, especialmente a grupos de estatus socio-económico bajo (Smith, Kendall y Keefe 2002), y la prioridad de descubrir cuáles son los componentes activos de los programas con la finalidad de reducir la duración de los mismos y mejorar la relación coste-beneficio.

El presente estudio es un intento de validar un programa de tratamiento que sigue de forma particular los procedimientos metodo- lógicos del grupo de Friedman como resultado de haber asistido en San Francisco a las reuniones de los grupos de participantes en el Coronary/Cancer Prevention Project. Nuestra perspectiva teórica, sin embargo, está más próxima a la que expone Roskies (1987).

\section{Objetivos}

El presente estudio intenta validar un programa de intervención psicológica para modificar conductas prono-coronarias, especialmente componentes del PCTA, en personas de un nivel social y educativo predominantemente bajos que han sufrido una angina de pecho y/o un infarto de miocardio. Los efectos positivos del tratamiento sobre las variables dependientes secundarias, ansiedad rasgo y depresión, contribuirá a la validación del tratamiento, aunque la ausencia de los mismos no invalidaría el tratamiento, puesto que el tratamiento no pretende directamente reducir ni la ansiedad ni la depresión.

Utilizando la estrategia de paquete de tratamiento (Kazdin y Wilson 1978), comenzamos elaborando un paquete de tratamiento cognitivo-conductual de amplio espectro, de modo que en una investigación posterior éste pueda desmantelarse.

\section{Método}

\section{Muestra y procedimiento de selección}

La muestra está compuesta por varones tratados en el Hospital Universitario de Canarias. El clima de esta región es suave, el estilo de vida en gran medida rural y la actividad económica más importante es la de los servicios relacionados con la industria del turismo. 
El ritmo de vida en las islas es, por tanto, más lento que en áreas industrializadas.

Todos los pacientes tratados en el Servicio de Cardiología entre los años 1992-1995 recibieron información por parte de un cardiólogo sobre la prevención secundaria de la EC y fueron invitados a participar en el proyecto si cumplían las tres condiciones siguientes: sufrir EC documentada de forma fiable, en la mayoría de los casos tras un cateterismo; no haber recibido la recomendación de someterse de forma inmediata a una intervención quirúrgica y no presentar ninguna comorbidad médica o psicológica que requiriese una intervención individual.

El proyecto se desarrolló en tres promociones o años. Al comienzo de cada año se organizaba una reunión con los 60 pacientes coronarios contactados en primer lugar que cumplieran los criterios de elegibilidad y hubieran expresado su interés en el proyecto mientras estaban en el hospital. En esta reunión se daba a los pacientes una pequeña explicación de las ventajas de participar en un Tratamiento Psicológico (TPs) o un Programa de Educación para la salud (PES) para prevenir la recurrencia de episodios coronarios. También se les explicaba la necesidad de contar con voluntarios par participar en un grupo de control que sólo recibiría, en principio, el Tratamiento Médico Estándar (TME) para pacientes coronarios. Además se les explicaba el calendario de los programas de tratamiento y las responsabilidades adquiridas por los participantes.

Después de esta reunión el equipo de investigación distribuyó a los participantes en diferentes grupos. Los 40 primeros en aceptar su participación en el proyecto fueron distribuidos al azar entre los grupos de TPs y PES y los 20 últimos, o los que tenían alguna dificultad en asistir a las sesiones de tratamiento en ese tiempo, fueron asignados al grupo de control con sólo el TME. Por tanto, el diseño de investigación constó de un grupo experimental que recibió el TME más el PES y otro que recibió sólo el TME. Este último grupo era de hecho un grupo de control en lista de espera, puesto que sus componentes tenían la posibilidad de participar en los grupos de tratamiento psicológico o educativo en los años siguientes del programa.

En una segunda reunión, los pacientes que seguían interesados en participar en el programa cumplimentaban un impreso con sus datos personales y firmaban su libre consentimiento en la participación y su conocimiento de los compromisos asumidos. En esta sesión las enfermeras y los psicólogos encargados de desarrollar los tratamientos y un médico que actuaba en representación del Hospital Universitario firmaban también las responsabilidades asumidas. Los participantes cumplimentaban además los tests psicológicos y los cuestionarios. Tras esta sesión, el psicólogo responsable del programa tenía una entrevista individual con cada participante para informarle sobre el grupo al que habían sido asignados.

En el primer año del proyecto los participantes en los grupos de TPs y PES fueron sorteados y distribuidos estrictamente al azar, pero las diferencias resultantes en cuanto a nivel educativo, en grupos de un nivel educativo bajo en general, hizo difícil desarrollar los programas de tratamiento. Por ello, en los años siguientes se decidió que la adscripción a los grupos se hiciera de forma estratificada. Se formaron dos grupos en función del nivel educativo y se les asignó al azar un programa de tratamiento. El tratamiento recibido por cada grupo se invirtió al año siguiente.

Cada año se contó con el mismo número de pacientes en el grupo de control con sólo el TME y en los grupos de tratamiento psicológico y educativo. El tamaño total de la 
muestra de participantes estuvo determinado exclusivamente por el número de pacientes dispuestos a participar, pues la información sobre el programa se dio a todos los pacientes varones del Servicio de Cardiología durante los tres años que duró el proyecto.

\section{Descripción y administración de la variable independiente}

El tratamiento psicológico resumido en del Pino (1998), consta de cinco partes: 1) Introducción a la EC y a sus factores de riesgo. 2) Control de la tensión, incluyendo a) Tensión física, particularmente mediante la práctica de la relajación; b) Tensión conductual, especialmente de urgencia del tiempo; c) Tensión cognitiva modificando los pensamientos disfuncionales; y d) Emociones negativas, principalmente ira-hostilidad y frustración, mediante la sustitución de estas por un pensamiento productivo y por emociones saludables. 3) Control del estrés trabajando en el reconocimiento de los disparadores personales de estrés y en cómo afrontar las diversas situaciones de estrés. 4) Planificación y disfrute del placer como una condición que permite reducir la tensión y el estrés. 5) El cambio del estilo de vida como un objetivo para toda la vida. Las indicaciones sobre el contenido de las sesiones y los trabajos para casa fueron recogidos en la Guía del Participante que se entregó a todos los componentes de los grupos de TPs. Todas las sesiones de tratamiento fueron dirigidas e impartidas por el primer autor de este artículo que contó con la colaboración de los tres mismos psicólogos durante los tres años del proyecto. Para asegurar la integridad y calidad del tratamiento se elaboró también una Guía del Terapeuta.

El programa de educación para la salud constó de cuatro módulos: 1) Anatomía y fisiología del corazón. 2) La EC: a) Manifestaciones clínicas y modalidades de tratamiento; b) Signos y síntomas de alarma; c) Factores de riesgo. 3) Estilos de vida recomendados incluyendo indicaciones sobre el cambio de dieta y de ciertos patrones de conductas prono-coronarias. 4) La medicación: sus efectos y dosis. El programa de educación para la salud fue impartido por un equipo de tres enfermeras que son profesoras de la Escuela de Enfermería de la Universidad de La Laguna.

Las sesiones de 90 minutos constaban de una exposición oral con la ayuda de material audio visual seguida de una fase de preguntas y respuestas. Al final, el encargado de dirigir la sesión presentaba el trabajo para casa que era revisado al comienzo de la sesión siguiente con la participación activa de los pacientes.

Los dos programas de tratamiento, el psicológico y el educativo, constaron de 24 sesiones desarrolladas a lo largo de nueve meses, 12 sesiones durante los tres primeros meses y otras 12 durante los seis meses siguientes. Después de finalizado el tratamiento hubo, en un segundo año, diez sesiones, una cada mes, para mantener los efectos del tratamiento. Los grupos estuvieron compuestos inicialmente de un número de personas que osciló entre un máximo de doce y un mínimo de ocho.

\section{Administración, descripción y evaluación de las variables dependientes}

Las variables dependientes fueron aplicadas en grupo por el primer autor de este artículo ayudado por un mínimo de tres colaboradores. La Entrevista Estructurada, necesariamente, fue aplicada de forma individual. 
El Inventario de Actividad de Jenkins (IAJ) (Jenkins, Zyzanski y Rosenman 1979) se aplicó utilizando la traducción experimental de Flores, Valdés y Sans (1982) y se evaluó de acuerdo con la propuesta de del Pino, Dorta y Gaos (1993). Esta propuesta evita las transformaciones injustificadas presentadas en el Manual del IAJ y ofrece una solución factorial de tres factores: Prisa-Impaciencia, Implicación en el trabajo y Comportamiento duro-Competitividad. Las respuestas se evaluaron utilizando una escala tipo Likert que va de dos a ocho puntos, de modo que las respuestas que indican un mayor acuerdo con el PCTA tengan las puntuaciones mayores.

La Entrevista Estructurada (EE) compuesta de tres factores (CompetitividadComportamiento duro, Ira manifiesta y Prisa-Impaciencia) se aplicó tal como se indica en del Pino, Gaos y Dorta (1999). Todas las entrevistas fueron realizadas por dos colaboradoras entrenadas y fueron evaluadas tras el último periodo de dos años de seguimiento por una persona que desconocía el proyecto de investigación y había sido entrenada especialmente para esta tarea.

La Ansiedad Rasgo fue evaluada mediante el Cuestionario de Ansiedad Estado-Rasgo (Spielberger, Gorsuch y Lushene, 1970) en la adaptación española de Seisdedos (1988).

La depresión fue evaluada utilizando la traducción española adaptada del Cuestionario de Depresión de Beck (CDB) (Beck et al. 1961) de Conde, Esteban y Useros (1976).

\section{Análisis de datos}

Los efectos del tratamiento fueron analizados mediante un Análisis de Varianza de Medidas Repetidas. Los resultados obtenidos tras el año de tratamiento responden a un diseño de $3 \times 2$, con un factor entre gru- pos con tres niveles (TPs, PES y TME) y un factor intra-grupo con dos tiempos de medida (antes y después del tratamiento). Los resultados obtenidos tras los dos años de seguimiento responden a un diseño de 2 x 4 con dos niveles en el factor entre-grupos (TPs y PES) y un factor intra-grupo, el tiempo de medida, con cuatro niveles, antes del tratamiento, tras concluir el año de tratamiento y uno y dos años después de haber concluido el tratamiento. Todos los análisis fueron realizados utilizando el SPSS 10.0.

\section{Resultados}

El proceso de selección de la muestra y los resultados de éste pueden verse en la Figura 1.

Como puede verse en el diagrama, alrededor del $50 \%$ de los pacientes que aceptaron participar en el programa mientras estaban en el hospital no acudieron a la primera reunión de presentación. El 12\% de los que asistieron a esta reunión dejaron de asistir a una segunda sesión en que los participantes dieron su consentimiento firmando las condiciones de participación.

La media de asistencia a las 24 sesiones del programa en el grupo de TPs fue de 20,64 , el $86 \%$ de las asistencias posibles, y la asistencia en el grupo de PES fue del 19,68 , el $82 \%$. El rango de asistencia varió entre 12 y 24 . Se consideraron abandonos en los grupos de tratamiento quienes no asistieron al 50\% de las reuniones. En el grupo de control con TME se consideraron abandonos quienes cumplimentaron los cuestionarios sólo al inicio del programa.

Los análisis de la distribución proporcional de las características demográficas y clínicas de los tres grupos (68 participantes) y de estos 
Figura 1

Proceso de selección y distribución de la muestra

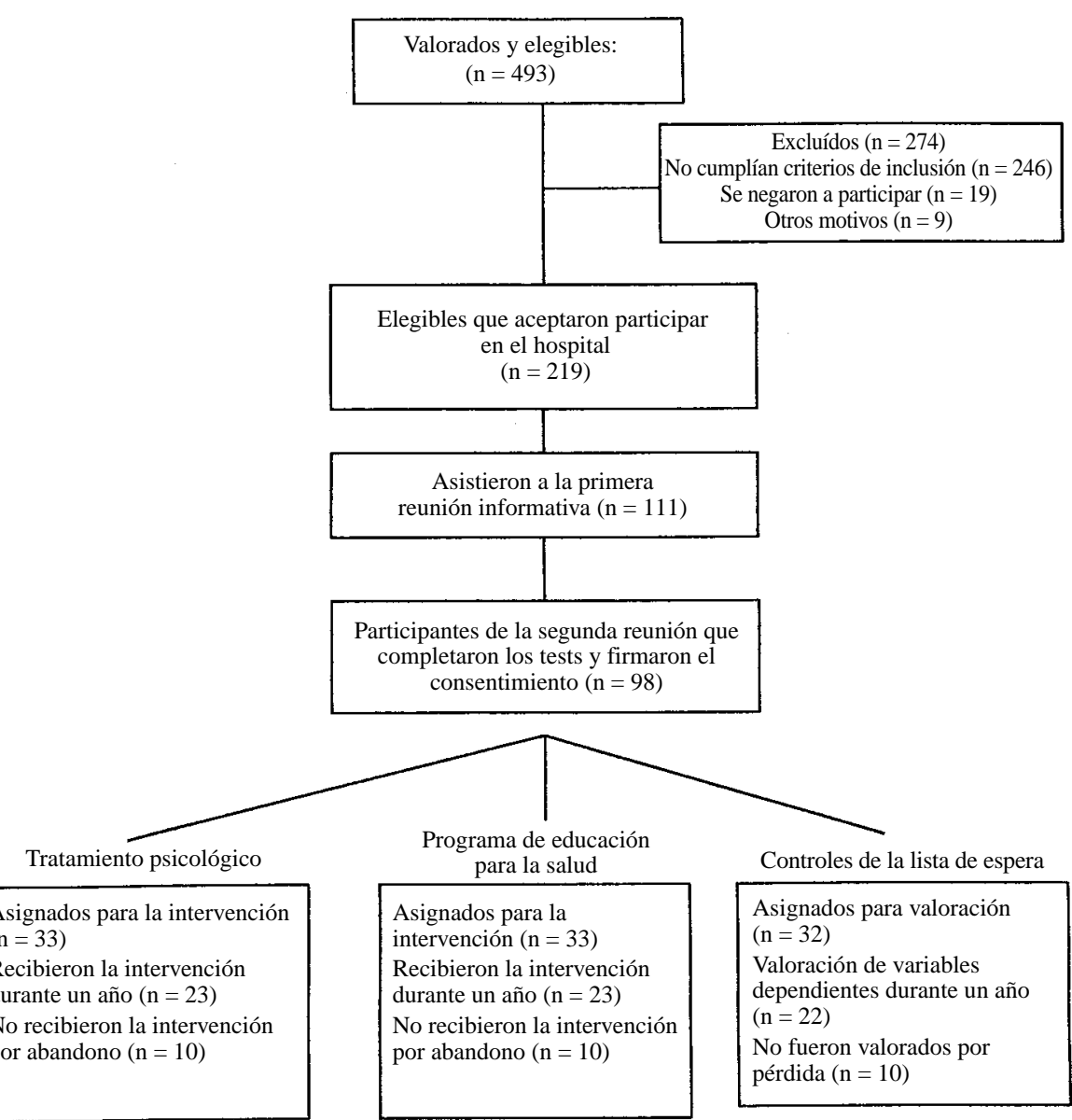

Seguimiento de 1 año $(\mathrm{n}=19)$

Seguimiento de 2 años $(\mathrm{n}=19)$

Perdidos en el seguimiento $(n=4)$

Motivos:

1 falleció

1 cambió de domicilio

2 se negaron a continuar

Seguimiento de 1 año $(\mathrm{n}=18)$ Seguimiento de 2 años $(n=18)$

Perdidos en el seguimiento $(n=5)$

Motivos:

2 fallecieron

2 cambiaron de domicilio

1 se negaron a continuar
Analizados después de:

1 año $(\mathrm{n}=23)$

2 años ( $\mathrm{n}=19)$
Analizados después de:

1 año $(\mathrm{n}=23)$

2 años $(\mathrm{n}=18)$
Sin seguimiento

Analizados después de:

1 año $(n=22)$

Sin seguimiento 


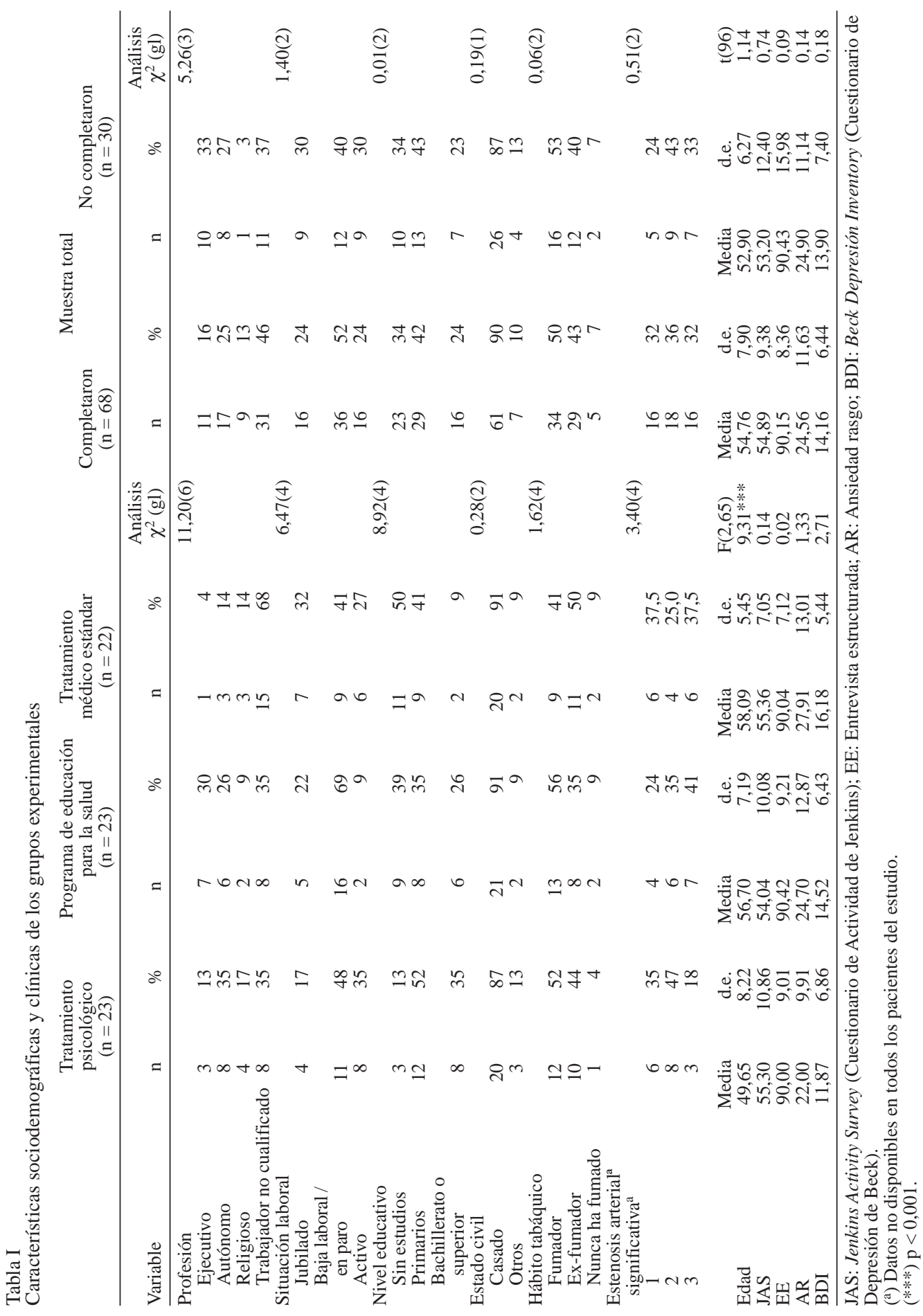


68 participantes comparados con los 30 casos de abandono pueden verse en la Tabla I.

Como puede apreciarse, los 68 componentes de los tres grupos son homogéneos en todas las variables excepto en la edad. El test de rangos de Duncan indica que el grupo que recibió TPs era más joven que los grupos que recibieron el PES y el TME, que no se diferencian entre sí en esta variable. La diferencia en edad de los grupos no estuvo asociada a diferencias estadísticamente significativas en las variables dependientes estudiadas.

Las comparaciones entre los 68 participantes de los diferentes grupos con asistencia regular y los 30 que abandonaron no mostraron diferencias significativas en ninguno de los descriptores de los grupos.

\section{Análisis tras el año de tratamiento}

Las medidas de tendencia central de los tres grupos antes y después del tratamiento y dos estadísticos, F y el Tamaño del Efecto (TE), de los contrastes intra-grupo pueden verse en la Tabla II.

Conducta Tipo A. En el ANOVA de medidas repetidas realizado sobre las puntuaciones del IAJ sólo los efectos del 'Tiempo de medida' presentan valores de $\mathrm{F}$ con una probabilidad inferior al 0,05 $[\mathrm{F}(1,65)=29,75$, p.<.001]. Esta reducción de la Conducta Tipo A queda reflejada en los tres grupos como puede verse en la Tabla II. Los contrastes entre los tiempos de medida realizados a partir de los factores del IAJ indican que las puntuaciones del grupo que recibió TPs se redujeron significativamente en el factor 1 , Prisa-Impulsividad $[\mathrm{F}(1,22)=7,80, p .=0,01$, $\mathrm{TE}=0,58]$, y en el factor 3, Comportamiento duro y Competitividad $[\mathrm{F}(1,22)=5,94, p$. $<$ $0,05, \mathrm{TE}=0,51]$. Las puntuaciones del grupo del PES se vieron reducidas significativamente también en el factor $1[\mathrm{~F}(1,22)=$ $14,02, p=0,001, \mathrm{~T}=0,78] \mathrm{y}$ en el factor 3 $[\mathrm{F}(1,22)=8,88, p .<.01, \mathrm{TE}=0,62]$, mientras que la reducción significativa en Conducta

Tabla II

Valores medios, desviaciones estándar y contrastes entre grupos antes y después del tratamiento

\begin{tabular}{lrrrrrrr}
\hline & & \multicolumn{2}{c}{ Pre-tratamiento } & \multicolumn{2}{c}{ Post-tratamiento } & \multicolumn{2}{c}{ Contrastes entre grupos } \\
& N & Media & d.e. & Media & d.e. & F & ES \\
\hline JAS & & & & & & & \\
TPs & 23 & 55,30 & 10,86 & 49,30 & 11,99 & $9,95^{* *}$ &, 66 \\
PES & 23 & 54,04 & 10,08 & 47,21 & 11,65 & $14,76^{* * *}$ &, 80 \\
TME & 22 & 55,36 & 7,05 & 50,27 & 9,54 & $6,40^{*}$ &, 54 \\
EE & & & & & & & \\
TPs & 22 & 90,00 & 9,01 & 84,18 & 11,45 & $8,24 * *$ &, 62 \\
PES & 21 & 90,42 & 9,21 & 88,24 & 10,44 & 1,56 &, 27 \\
TME & 22 & 90,04 & 7,12 & 88,18 & 7,81 & 2,17 &, 31 \\
AR & & & & & & & \\
TPs & 23 & 22,00 & 9,91 & 17,00 & 8,84 & $17,09 * * *$ &, 86 \\
PES & 23 & 24,70 & 12,87 & 21,52 & 9,04 & 3,42 &, 38 \\
TME & 21 & 27,91 & 13,01 & 23,91 & 12,65 & 2,22 &, 32 \\
BDI & & & & & & & \\
TPs & 23 & 11,87 & 6,86 & 9,70 & 4,76 & 3,82 &, 41 \\
PES & 23 & 14,52 & 6,43 & 14,35 & 6,13 & 0,28 &, 03 \\
TME & 22 & 16,18 & 5,44 & 13,32 & 4,32 & 5,65 &, 51 \\
\hline
\end{tabular}

JAS: Jenkins Activity Survey (Cuestionario de Actividad de Jenkins); EE: Entrevista estructurada; AR: Ansiedad rasgo; BDI: Beck Depresión Inventory (Cuestionario de Depresión de Beck).

TPs: Tratamiento psicológico; PES: Programa de educación para la salud; TME: Tratamiento médico estándar.

$* \mathrm{p} \leq 0,05 ; * * \mathrm{p} \leq 0,01 ; * * * \mathrm{p} \leq 0,001$. 
Tipo A global del grupo de control que sólo recibió el TME no aparece al analizar los factores que componen la escala total.

La Escala Total de la EE mostró efectos significativos en el ANOVA al nivel de confianza del 0,05 sólo en la fuente de variación 'Tiempo de medida' $[\mathrm{F}(1,62)=11,10, p=$ $0,001]$. Los contrastes entre tiempos de medida realizados por grupos indican que sólo en el grupo que recibió el TPs se produjo una reducción significativa de la Conducta Tipo A, como puede verse en la Tabla II. Esta reducción aparece sólo en el factor 3 de la EE, Prisa-Impaciencia $[\mathrm{F}(1,21)=10,79$, $p<0,001, \mathrm{~T}=0,70]$.

Ansiedad-Rasgo. Sólo la fuente de variación 'Tiempo de medida' ofreció valores de F estadísticamente significativos para los ANOVA de medidas repetidas, $[\mathrm{F}(1,64)=$ 13,40, $p=0,001]$. Al analizar los efectos significativos por grupos sólo se apreció una diferencia altamente significativa en el grupo de TPs, como aparece en la Tabla II.

Depresión. Los resultados del ANOVA presentaron efectos significativos en la fuente de variación "Tiempo de medida" $[\mathrm{F}(1,65)=7,27, p<0,01]$ y "Grupo" $[\mathrm{F}(2,65)=4,27, p<0,05]$. La diferencia significativa entre los tiempos de medida se resume sólo en una reducción significativa de las puntuaciones en el CDB de los componentes del grupo de TME, como puede verse en la Tabla II. La diferencia entre grupos se refleja, especialmente, en una diferencia significativa entre los componentes de los grupos de TPs y del PES después del tratamiento $[\mathrm{F}(2,65)=5,18, p<0,01]$.

\section{Análisis tras dos años de seguimiento}

Conducta Tipo A. En los resultados de la Escala Total del IAJ, el ANOVA de medidas repetidas presentó efectos significativos sólo en la fuente de variación "Tiempo de medida" $[\mathrm{F}(3,105)=8,90, p<0,001]$. Este efecto se basa en los cambios en el factor 1, PrisaImpulsividad, $[\mathrm{F}(3,105)=9,72, p<0,001]$, en el que los grupos de TPs y del PES redujeron las puntuaciones en todos los contrastes intragrupo y en el factor 3, Perseverancia-Competitividad, $[\mathrm{F}(3,105)=5,93, p=0,001]$, en el que sólo el grupo de TPs redujo sus puntuaciones en todos los contrastes intra-grupo. Estos contrastes intra-grupo muestran, como puede verse en la Tabla III, que las puntuaciones en la Escala Total del IAJ disminuyeron tras el tratamiento y se mantuvieron tras un año de seguimiento en ambos grupos. Sin embargo, en el grupo que recibió TPs la reducción entre los tiempos de medida 1-3 y 1-4 se incrementó respecto al contraste entre los tiempos de medida 1-2, mientras que en el grupo del PES la diferencia significativa entre los tiempos de medida 1-2 se redujo en el contraste tras un año de seguimiento y desapareció tras un seguimiento de dos años.

Los resultados de la Escala Total de la EE muestran que sólo el 'Tiempo de Medida' determina la aparición de efectos significativos $[\mathrm{F}(3,105)=2,92, p<0,05]$. Los contrastes entre tiempos de medida por factores y grupos indican que en el Total de la Escala se dieron contrastes significativos entre el tiempo de medida 1, antes del tratamiento, y todos los otros tiempos de medida sólo en el grupo que recibió TPs, como aparece en la Tabla III. Estos cambios tuvieron lugar en el factor 3, Prisa-Impaciencia, que disminuyó significativamente después del tratamiento $[\mathrm{F}(1,18)=7,54, p<0,01]$ y estos cambios se mantuvieron en el primer y segundo año de seguimiento $[\mathrm{F}(1,18)=4,63, p<0,05 ; \mathrm{y}$ $9,49, p<0,01$, respectivamente].

Ansiedad-Rasgo. En esta variable se dieron efectos significativos en la fuente de variación 'Tiempo de Medida' $[\mathrm{F}(3,105)=$ 
244 ANTONIO DEL PINO ET AL.

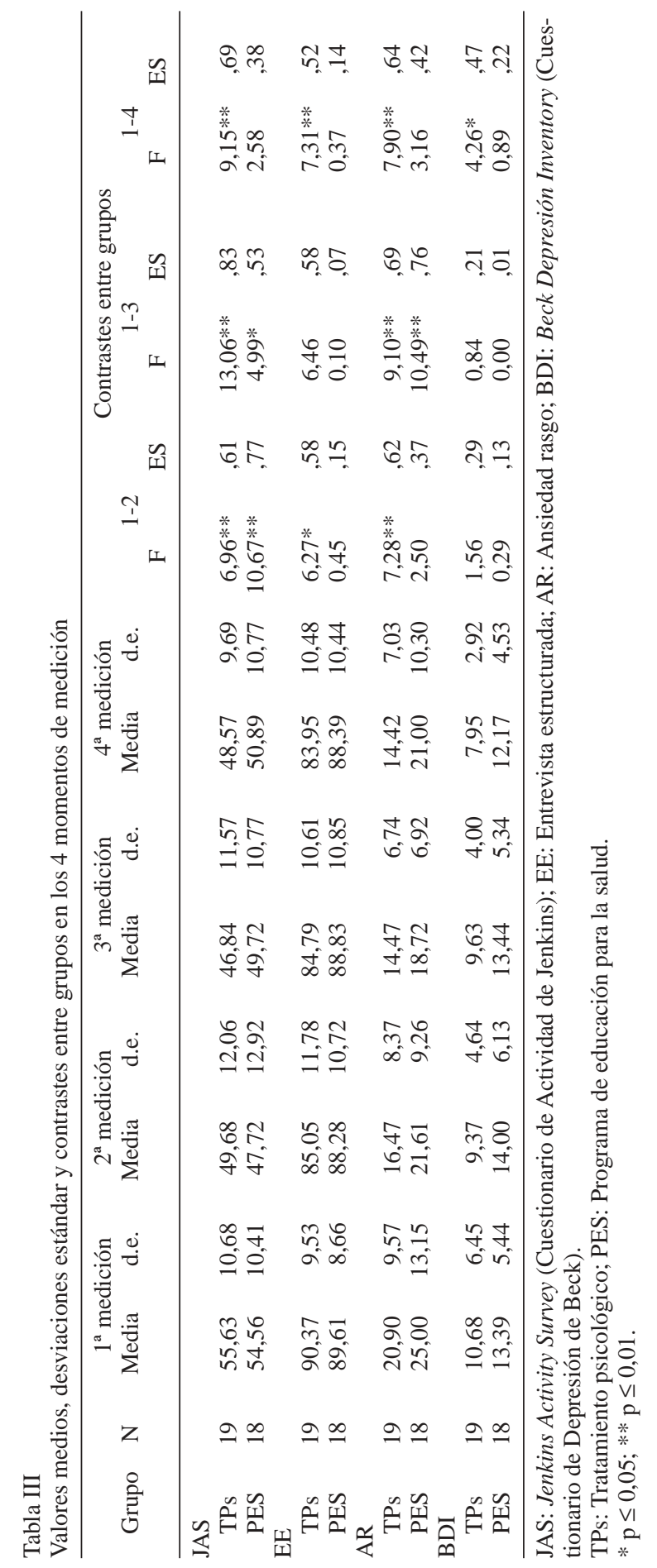


$8,88, p<0,001]$. Los contrastes por grupos entre tiempos de medida que pueden verse en la Tabla III mostraron una reducción al nivel de significación del 0,01 entre los tiempos de medida $1-2,1-3$ y $1-4$ en el grupo que recibió TPs. En el grupo del PES apareció también una diferencia significativa al mismo nivel de confianza en el contraste entre los tiempos de medida 1-3.

Depresión. El ANOVA indicó sólo un efecto significativo que tiene al 'Grupo' como fuente de variación $[\mathrm{F}(1,35)=8,58, p<$ 0,01]. El análisis posterior de las diferencias indica que los grupos no difieren entre sí en depresión al nivel de confianza del 0,05 antes del tratamiento, $[\mathrm{t}(35)=1,37]$, pero en los tres tiempos de medida siguientes el grupo de TPs puntuó significativamente menos en depresión que el grupo que recibió el PES, $[\mathrm{t}(35)=2,60, p<0,05 ; 2,47, p<0,05 ; \mathrm{y} 3,39$, $p .<0,01$ para las comparaciones entre grupos en los tiempos de medida 2, 3 y 4 respectivamente]. La progresiva reducción de las puntuaciones en el CDB en el grupo de TPs queda reflejada en una diferencia significativa intra-grupo entre los tiempos de medida 14, como puede verse en la Tabla III.

\section{Discusión y conclusiones}

La característica distintiva de este estudio es que se ha desarrollado con pacientes coronarios varones de nivel socio-económico bajo, un grupo escasamente representado en los programas de intervención (Smith, Kendall y Keefe 2002). Puesto que éste es un estudio en el que los criterios de participación no fueron restrictivos, creemos que los resultados obtenidos podrían extenderse a todos los pacientes varones con EC que viven en las Islas Canarias y reciben atención sanitaria en el servicio público de salud. Sin embargo, esta generalización podría modularse en aten- ción al porcentaje de pacientes que rechazan participar cuando se les invita a ello durante el periodo de hospitalización, (7,98\%), y al alto porcentaje de pacientes que, después de haber aceptado participar en el programa durante el periodo de hospitalización, o no asisten a la primera reunión de grupo $(49,31 \%)$ o, aunque asisten a la primera reunión, no participan en la segunda en que se hace firme la participación en el programa (5,94\%). Billings et al. (1996) también informan de un rechazo a participar en su estudio en torno al $50 \%$. Nosotros atribuimos estos resultados a la falta de información sobre los efectos positivos de estos programas sobre la salud y calidad de vida de los pacientes y a la falta de implicación en estos programas por parte del equipo médico. Sin embargo, también podrían influir factores sociales y económicos, puesto que los participantes con menos asistencia a las reuniones de grupo fueron los de niveles educativos y económicos considerablemente más bajos, dicho esto respecto a un grupo que ya viene definido por niveles educativos y económicos bajos.

La tasa de abandonos durante el año de tratamiento apenas alcanza el $31 \%$, un porcentaje más bajo que el que se produce en los estudios basados en la población (Chambless y Ollendick 2001) y prácticamente idénticos al que se da en los estudios sobre efectividad de los tratamientos desarrollados con grupos especialmente controlados (Elkin et al. 1989). Kazdin (1996) informa que la tasa de abandonos en la terapia de adultos es del 40\%-60\%, aunque la evaluación de los abandonos debería analizarse tomando en consideración los parámetros utilizados para definir los casos de abandono, la duración del tratamiento, las compensaciones marginales recibidas dentro y fuera del tratamiento y otros aspectos de esta naturaleza.

Estos resultados sobre la participación indican que el diseño y desarrollo del trata- 
miento responden, en gran medida, a las necesidades y expectativas de los participantes. En nuestro caso consideramos que la duración del tratamiento fue apropiada, porque nos enfrentamos a un estilo de vida que se desarrolla a lo largo de muchos años, invade todos los ámbitos de la vida de una persona y se basa en los aspectos más íntimos de la personalidad (Friedman 1996).

Las características socio-educativas y de salud de los participantes son muy homogéneas. La única diferencia entre grupos en los parámetros demográficos y culturales fue la menor edad de los componentes del grupo de TPs, lo que implicaría la probabilidad de que este grupo antes del tratamiento mostrara mayor presencia del PCTA (Dielman, Butchard y Moss, 1990), dando, de este modo, mayor relevancia a la reducción de los componentes del PCTA que se consigue en este grupo.

Los resultados tras el año de tratamiento indican que el TPs tiene efectos específicos sobre la conducta prono-coronaria, porque los cambios más claros y consistentes tuvieron lugar en el grupo que recibió este tratamiento y estos cambios se verificaron no sólo en los contrastes con el grupo de control con sólo el TME, sino también en contraste con el grupo de control que recibió el PES. El grupo del PES, tras la fase de tratamiento, sólo presentó una diferencia significativa en Prisa-Impulsividad, un factor del IAJ que es un autoinforme, pero no en Prisa-Impaciencia, un factor similar de la EE. Los diferentes resultados del grupo del PES en el IAJ y en la EE podrían estar reflejando el hecho de que durante el programa se reseñan los efectos indeseables del PCTA, de modo que los participantes evaluados mediante auto-informes tienden a negar tras la fase de tratamiento la presencia de tal conducta en sus vidas. En la EE, en que los participantes responden directamen- te al entrevistador, es menos probable que controlen sus respuestas y nieguen la presencia del PCTA. Por ello, es recomendable utilizar la EE para evaluar los resultados psicológicos de estos tratamientos. Los grupos que recibieron el PES tenían información sobre el PCTA porque en el contenido de su programa se contemplaba una charla sobre el PCTA y porque eran conocedores de lo que se hacía en los grupos de TPs.

El tamaño del efecto de los cambios en la Escala Total de la EE, 0,62, y en el IAJ, 0,82, obtenido en el grupo que recibió TPs fue mayor que la media del tamaño del efecto 0,61 , obtenido en los estudios incluidos en el meta-análisis de Nunes, Frank y Kornfeld (1987). Es importante reseñar los resultados obtenidos con la EE, porque su relación con la EC se ha demostrado de forma consistente y permite la evaluación del estilo de las respuestas y no sólo el contenido de las mismas.

Los resultados de la fase de seguimiento corroboran los obtenidos tras el año de tratamiento. Los cambios que se produjeron en el factor 1 del IAJ, Prisa-Impulsividad, son más evidentes en el grupo que recibió TPs, y los cambios en la EE que aparecieron sólo en el grupo de TP se mantienen en las diferentes comparaciones entre los tiempos de medida, incluyendo las realizadas tras dos años de seguimiento. Estos cambios son congruentes con las sesiones específicamente diseñadas en estos grupos para reducir la tensión conductual controlando la urgencia del tiempo y dominando la prisa.

Los resultados obtenidos en las variables dependientes secundarias corroboran en cierta medida la validez interna del tratamiento. Los resultados en Ansiedad Rasgo muestran cambios significativos tras el tratamiento sólo en el grupo de TPs, cambios que continúan apareciendo en la fase de seguimiento hasta el último tiempo de medida. En el grupo del PES sólo aparecen diferencias significativas 
cuando se comparan las puntuaciones iniciales con las obtenidas en el tercer tiempo de medida, un año después de haber concluido el tratamiento. Los resultados en el CDB indican que tras el tratamiento sólo el grupo de control que recibió TME mostró una reducción significativa en depresión. Este cambio, que podría parecer sorprendente, pensamos que podría considerarse una regresión a la media de los grupos, puesto que antes del tratamiento la puntuación del grupo TME era superior, 16,41, a la de los grupos que recibieron TPs, 11,87, o el PES, 14,52. Después del año de tratamiento, el grupo de TME obtuvo una puntuación en depresión de 13,32, aproximadamente la media de los tres grupos antes y después del tratamiento, 14,23 y 12,44 , respectivamente. Después del tratamiento el grupo que recibió TPs no presenta cambios intra-grupo estadísticamente significativos, pero sus puntuaciones iniciales fueron reduciéndose tras el tratamiento y continuó decreciendo durante los dos años de seguimiento hasta que al final aparece un cambio estadísticamente significativo entre los tiempos de medida 1-4. Esta reducción progresiva de las puntuaciones en depresión queda reflejada en las diferencias significativas entre los grupos de TPs y del PES en los tiempos de medida 2, 3 y 4, aunque ambos grupos no difieren en depresión antes del tratamiento. Las reducciones observadas en las variables dependientes secundarias parecen depender en primer lugar de la modalidad de tratamiento, porque el grupo de TPs, que recibió orientaciones específicas para cambiar los pensamientos disfuncionales y las emociones nocivas y sustituirlas por pensamientos productivos y emociones saludables, es el único que presentó tras el tratamiento una reducción en ansiedad y una reducción progresiva en depresión a través de las diferentes fases. En segundo lugar, estas reducciones observadas podrían deberse al paso del tiempo, puesto que el grupo del PES, que no pre- senta cambios tras el tratamiento ni en depresión ni en ansiedad, muestra un cambio significativo en ansiedad en la fase de seguimiento. Esta hipótesis podría haberse confirmado si hubiéramos tenido también un seguimiento de dos años en el grupo de control con el TME. Esto no fue posible, sin embargo, puesto que algunos de los participantes en este grupo quisieron ser incluidos en los grupos con tratamiento adicional y otros no quisieron continuar colaborando.

Para comprender los resultados $\mathrm{y}$, en general, la discusión final es importante indicar que alrededor de un $20 \%$ de los participantes, cuatro en el grupo de TPs y cinco en el grupo del PES, que figuran en los análisis referidos a post-tratamiento, no figuran por distintas razones en los análisis referidos a la fase de seguimiento.

En conclusión, consideramos que el tratamiento cognitivo-conductual ha sido capaz de modificar los componentes más conductuales del PCTA como la prisa y la impaciencia, que se recogen en el factor 3 de la EE y en el factor 1 del IAJ y que son especialmente nocivos según Friedman (1996), así como las puntuaciones de la Escala Total de la EE y del IAJ que vienen a ser medidas globales del PCTA. Las puntuaciones referidas a ansiedad y depresión se modificaron también en el grupo de TPs. La modificación en este grupo de la ansiedad y, sobre todo, de la depresión desde puntuaciones que pueden considerarse críticas hasta niveles de práctica normalidad no es usual en los programas psico-educativos, como se concluye en el meta-análisis de Dusseldorf et al. (1999), y puede considerarse un indicador de la mejora de la calidad de vida de los pacientes. Todos estos resultados corroboran la validez de este tratamiento cognitivo-conductual de amplio espectro y su posible uso y adaptación a las características de los pacientes coronarios de nivel socioecónomico bajo. 


\section{Agradecimientos}

Este estudio fue financiado por el Gobierno Autónomo de Canarias.

\section{Bibliografía}

BECK, A.T., WARD, C.H., MENDELSON, M., MOCK, J., ERBAUGH, J. An inventory for measuring depression. Archives of General Psychiatry, 4, 561-571, 1961.

BILLINGS, J.H., SCHERWITZ, L.W., SULLIVAN, R., SPARLER, S., ORNISH, D.M. The Lifestyle Heart Trial: Comprehensive treatment and group support therapy (pp. 233-253). In: Allan, R. \& Scheidt, S. (Eds.) Heart and Mind. Washington, DC: American Psychological Association, 1996.

CHAMBLESS, D.L., OLLENDICK, TH. Empirically supported psychological interventions: Controversies and Evident. Annual Review of Psychology, 52, 685-716, 2001.

CONDE, V., ESTEBAN, T., USEROS, E. Revisión crítica de la adaptación castellana del cuestionario de Beck. Revista de Psicologia General y Aplicada, 31, 469-497, 1976.

DIELMAN, T.D., BUTCHART, A.T., MOSS, G.E. Demographic differences in the relationships among components and global ratings of structured interview assessed type A behavior in a population sample. In: Strube, M.J. (Dir.), Type A behavior (Special Issue), Journal of Social Behavior and Personality, 5, 233-261, 1990.

DEL PINO, A. Tratamiento cognitivo-conductual para modificar el patrón de conducta tipo A (pp. 359-383). In: Caballo, V.E. (Dir.), Manual para el tratamiento cognitivoconductual de los trastornos psicológicos. Vol. 2. Madrid: Siglo Veintiuno de España Editores, S.A, 1998.

DEL PINO, A., DORTA, R., GAOS, M. El Inventario de Actividad de Jenkins (JAS). I. Análisis descriptivo y factorial. Boletín de Psicología, 41, 57-90, 1993.

DEL PINO, A. , GAOS, M.T., DORTA, R. Construct and criterion-oriented validity of the structured interview for the assessment of the type A behavior pattern in a sample of Canarian males. European Journal of Psychological Assessment, 15, 39-48, 1999.

DUSSELDORP, E., VAN ELDEREN, T., MAES, S., MEULMAN, J., KRAAIJ, V. A meta-analysis of psychoeducational programs for coronary heart disease patients. Health Psychology, 18, 506-519, 1999.

ELKIN, I., SHEA, T., WALKINS, J., IMBER, S., SOTSKY, S., COLLINS, J., GLASS, D., PILKONIS, P., LEBER,
W., DOCHERTY, J., FIESTER, S., PARLOFF, M. National Institute of Mental Health treatment of depression collaborative research program: General effectiveness of treatments. Archives of General Psychiatry, 46, 971-982, 1989.

FLORES, T. DE, VALDÉS, M., SANS, S. Inventario de Actividad de Jenkins (JAS). Forma C. Madrid: TEA Ediciones, S.A. (Experimental edition), 1982.

FRIEDMAN, M. Type A behavior: Its diagnosis and treatment. New York: PlenumPress, 1996.

JENKINS, C.D., ZYZANSKI, S.J., ROSEUMAN, R.H. Jenkins Activity Survey Manual. Form C. New York: Psychological Corporation, 1979.

KAZDIN, A.E. Dropping out of child psychotherapy: Issues for research and implications for practice. Clinical Child Psychology and Psychiatry, 1, 133-156, 1996.

KAZDIN, A.E., WILSON, G.T. Evaluation of behavior therapy: Issues, evidence, and research strategies. Cambridge, MA: Ballinger Publishing Company, 1978.

LINDEN, W., STOSSEL, C., MAURICE, J. Psychosocial interventions for patients with coronary artery disease. A meta-analysis. Archives of Internal Medicine, 156, 745752, 1996.

NUNES, E.V., FRANK, K.A., KORNFELD, D.S. Psychologic treatment for the type A behavior pattern for coronary heart disease: A meta-analysis of the literature. Psychosomatic Medicine 48, 159-173, 1987.

ROSKIES, E. Stress Management for Healthy Type A's. New York: The Guilford Press, 1987.

SEISDEDOS, N. Cuestionario de Ansiedad EstadoRasgo. Madrid: TEA Ediciones, S.A, 1988.

SMITH, TH. W., KENDALL, PH.C., KEEFE, F.J. Behavioral medicine and clinical health psychology: Introduction to the special issue. A view from the decade of behavior. Journal of Consulting and Clinical Psychology 70, 459-462, 2002.

SPIELBERGER, C.D., GORSUCH, R.L., LUSHENE, R.E. State-Trait Anxiety Inventory (Self Evaluation Questionnaire). Palo Alto, CA: Consulting Psychologists Press, 1970.

Dirección para correspondencia:

Antonio del Pino Pérez

Profesor, Departamento de Personalidad, Evaluación

y Tratamiento Psicológico

Facultad de Psicología

Universidad de La Laguna

Campus de Guajara

38205 La Laguna

SPAIN

E-mail: apino@ull.es 